\title{
EDAS/COAS Based Antenna Selection for Code Index Modulation Aided Spatial Modulation
}

\author{
Erdoğan Aydın \\ Department of Electrical Electronics Engineering, Medeniyet University, İstanbul, Turkey
}

Cite this article as: Aydın E. EDAS/COAS Based Antenna Selection for Code Index Modulation Aided Spatial Modulation. Electrica, 2019; 19(2): 113-119.

\begin{abstract}
In this study, a new code index modulation (CIM) aided spatial modulation (SM) technique based on the Euclidean distance and capacity optimized based antenna selection (EDAS/COAS), called EDAS/COAS-CIM-SM, is proposed for multiple-input multiple output (MIMO) systems. Since in the proposed EDAS/COAS-CIM-SM technique, incoming information data bits indicates modulated symbols, activated transmit antenna indices as well as their corresponding spreading code indices, data bits are conveyed not only by the modulated symbols but also by the indices of the active antenna and spreading code indices. Thus, the proposed two systems provide faster data rates while spending less transmission power and possessing better error performance than the conventional direct sequence- spread spectrum (DS-SS), SM, EDAS and COAS based SM (EDAS-SM, COAS-SM), and CIM based SS (CIM-SS) systems. In addition, in order to improve the overall performance of the CIM aided SM (CIM-SM) system, EDAS and COAS techniques are considered at the receiver. Computer simulations have also shown that the EDAS/COAS-CIM-SM system has better error performance than the CIM-SM system over the Rayleigh fading channels for binary phase shift keying (BPSK) modulation schemes.
\end{abstract}

Keywords: Spatial modulation, euclidean distance and capacity optimized based antenna selection, code index modulation, MIMO systems, direct sequence spread spectrum

\section{Corresponding Author: \\ Erdoğan Aydın}

E-mail:

erdogan.aydin@medeniyet.edu.tr

Received: 04.02.2019

Accepted: 19.02.2019

DOI: 10.26650/electrica.2019.19003

Content of this journal is licensed under a Creative Commons Attribution-NonCommercial 4.0 International License.

\section{Introduction}

The use of mobile data is growing exponentially in today's world. Concepts such as high-quality video conferencing, high-quality video and audio broadcasting, online gaming, sending and uploading large files, very low latency, need for low power consumption, and the necessity to connect to the internet anywhere and anytime are becoming more and more popular. According to experts' estimates, global internet traffic will exceed 71 Exabytes in 2022 [1]. Therefore, the evolution of new high data-rated, high energy \& spectral efficient communication systems has become mandatory. In order to meet the above mentioned needs, many new research topics such as Millimeter Wave (mmWave) communication, Spectrum Sharing, Beamforming, Device-to-Device communication (D2D), Moving Networks (MN), Ultra-dense Networks (UDN), Ultra-reliable Networks (URN), Internet of Things (IoT) Communications, Index Modulation (IM), multiple-input multiple-output (MIMO) are being studied [2, 3].

In order to optimize signal performance, MIMO technique has been studied in many new works. As its name suggests, MIMO structure is based on multi-antenna technology and is a rational technology that increases performance parameters such as data rate, spectral efficiency, and energy efficiency [4]. Spatial Modulation (SM) is one of the most popular methods recently studied in the field of MIMO systems. The SM method utilizes a single radio frequency (RF) chain during the same time period, as well as the advantages of multiple antennas. As a result of the use of a single RF chain, signal processing, and circuit complexity are greatly reduced while energy efficiency increases. In the SM scheme, a single transmitter antenna is selected in each transmission time slot and the extra information is carried on without having to pay any extra cost by detecting which antenna is selected on the receiving side. In other words, antenna indices also contribute the data transmission. The most important features of the SM 
are the multiplication gain increasing in proportion to the logarithm of the number of antennas, the reduction of the transmitter complexity by using a single RF chain, the reduction of power consumption, the reduction of the processing complexity of the receiver, the synchronization and the complete elimination of inter-channel interference (ICI) problems [5].

Antenna selection technique is another scheme in MIMO systems that communicate using a single transmitter RF chain as in SM. In the antenna selection techniques, the antenna with the best channel condition is selected by using the Channel State Information (CSI) and then the transmission is carried out via this antenna. Since communication is made over a single antenna throughout a symbol duration, $\mathrm{ICl}$ is eliminated, power consumption and complexity are greatly reduced $[6,7]$. The antenna selection techniques that have been of interest in the SM scheme for the last few years are both Euclidean Distance optimized Antenna Selection (EDAS) and Capacity Optimized Antenna Selection (COAS) based techniques, both of which greatly improve performance. There- fore, these techniques will be included in this study in order to increase performance much more. In simple terms, in the EDAS scheme, all possible vector sets are determined and then a specific set of antennas that maximizes the minimum Euclidean distance is found after that the communication is carried out with these antennas. On the other hand, in the COAS scheme, the best antenna selection is found by selecting the antenna that corresponds to the largest channel norms $[8,9]$. In addition, the EDAS system is applied to the cooperative-SM scheme in [10].

Over the past few years, there has been a great deal of interest in $\mathrm{IM}$, which is one of the most important research topics of $5 \mathrm{G}$ communications. As the name suggests, IM schemes transmit the indices of some medium such as antennas, RF mirrors, sub-carriers, spreading codes, modulation types, antenna activation sequences, etc. embedded in the signal. Thanks to this simple and rational scheme, extra information is transmitted using little or even no energy, spectral efficiency increases, and no additional hardware cost is required [11, 12].

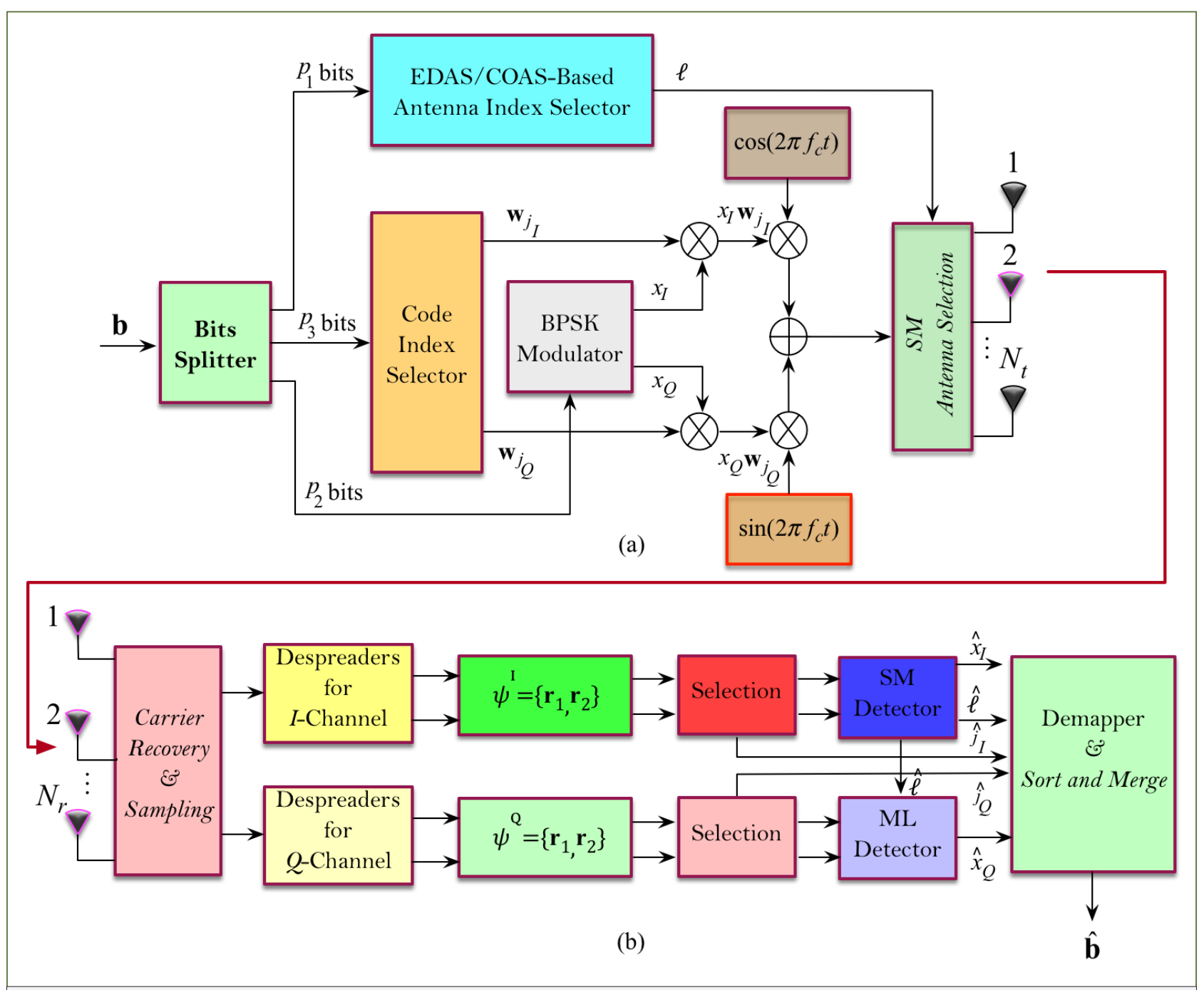

Figure 1. a, b. EDAS/COAS-CIM-SM system model, (a) transmitter, (b) receiver 
The IM technique used in this article is the code index modulation aided spread spectrum (CIM-SS) technique, which is considered among the competitors of the SM method. The CIM-SS technique is based on the direct sequence-spread spectrum (DS-SS) method, which is based on sending bit sequences to the receiver side by multiplying them with pseudorandom noise (PN) or spreading codes, thereby resisting noise and jamming. In the CIM-SS method, the extra information bits are transmitted over the orthogonal in-phase and quadrant (IQ)-channels by multiplying with spreading codes to the receiver side and this process is called "spreading". On the receiver side, the received signal is multiplied again with the spreading codes and is estimated by the correlators and this process is called "despreading". Since the extra bits of information are embedded into the spreading code indices, extra information bits are detected without any additional cost by detecting the spreading code. As a result, the CIM-SS method is a fast and secure communication technique because it hosts the advantages of the DS-SS method, transmits information via index modulation, and uses orthogonal channels for information transmission [13, 14]. More than these, a new high data-rated technique called CIM aided SM (CIM-SM), which is a combination of CIM-SS and SM methods, has been introduced at $[15,16]$ to the literature. The CIM-SM technique is faster, more spectral and energy efficient than the above-mentioned techniques.

In this study, new antenna selection-based MIMO transmission systems with high energy efficiency, high data rate and good error performance, called EDAS/COAS-CIM-SM, is proposed by combining EDAS, COAS, CIM, and SM techniques which are promising in $5 \mathrm{G}$ and beyond communication systems. It is shown via computer simulations that the proposed system performs data transmission at a higher rate, consumes less transmission energy, and also has better spectral efficiency and performance than the DS-SS, CIM-SS, SM, CIM-SM, EDAS-SM and COAS-SM techniques.

\section{System Model}

The general structure of the proposed EDAS/COAS-CIM-SM system model is shown in Figure 1. The considered system consists of the number of $N_{t}$ transmitting antennas, $N_{r}$ receiving antennas, and $N_{c}=2$ two different Walsh Hadamard spreading codes, i.e., $\boldsymbol{w}_{j}=\left[w_{j, 1}, w_{j, 2}, \ldots, w_{j, L}\right]^{T}, j \in\{1,2\}$ are used for the spreading operation. Also, each of spreading codes consists of $L$ chips. $N_{S M}$ out of $N_{t}$ antennas $\left(N_{S M} \leq N_{t}\right)$ refers to the number of antennas which will be activated by the EDAS/ COAS technique, and thus, $A=\{\ell\}_{\ell=1}^{N_{S M}}$ expresses the transmission antenna index set. Since EDAS/COAS based antenna selection technique is considered at the transmitter side, the performance of the SM receiver with the EDAS/COAS is better than the traditional SM receiver. Therefore, not only the active antenna index and symbol estimation performance on the receiving side will be improved compared to traditional SM scheme, but also make better the performance of the code index detection compared to CIM-SS and CIM-SM techniques. Binary phase shift keying (BPSK) modulation is envisaged for the proposed system. IQ-channel components are used both in the transmitter and receiver sides. It is assumed that the CSI is known at the transmitter and receiver side perfectly. Finally, the considered system structure is designed by adapting EDAS, COAS, SM, CIM techniques and BPSK modulation.

As seen from the transmitter structure of the EDAS/COASCIM-SM scheme depicted in Figure 1 is the incoming binary information data bits to be transmitted through one symbol period $\left(T_{S}\right)$. In the considered scheme, $\mathbf{b}$ is divided into $p_{1}$ $=\log _{2}\left(N_{S M}\right), p_{2}=\log _{2}(2 M)$, and $p_{3}=\log _{2}\left(2 N_{C}\right)$ sub-vector groups such that $p=p_{1}+p_{2}+p_{3}$. The $p_{1^{\prime}} p_{2}$, and $p_{3}$ sub-block groups of bits are transmitted in the antenna indices of SM, BPSK symbols and indices of spreading codes, respectively. $x_{1}$ and $x_{Q} \epsilon \pm 1$ BPSK symbols of the $I Q$-components are determined according to the bits sequence of $p_{2}$, The $x_{1}$ and $x_{Q}$ are then spreaded by the $\boldsymbol{w}_{j l}$ and $\boldsymbol{w}_{j Q}$ spreading code selected by the bits sequence of $p_{3}$. Finally, spreaded signals $x_{1} \boldsymbol{w}_{j 1}$ and $x_{Q}$ $\boldsymbol{w}_{j Q}$ are transmitted through $I Q$-components from the $I^{\text {th }}$ antenna activated by bits sequence of $p_{l}$. From this point forth, the faded noisy signal received by the EDAS/COAS-CIM-SM receiver can be expressed as follows:

$$
\begin{aligned}
r(t) & =\sum_{l=1}^{L}\left(x_{I} w_{j_{I}} g\left(t-l T_{c}\right) \cos \left(2 \pi f_{c} t\right)\right. \\
& \left.+x_{Q} w_{j_{Q}} g\left(t-l T_{c}\right) \sin \left(2 \pi f_{c} t\right)\right) h(t)+n(t),
\end{aligned}
$$

where, $g(t)$ is the unit rectangular pulse shaping filter on $[0, T]$ period time, here $T_{c}$ is the chip duration of the spreading code. $f_{c}$ is the carrier frequency. $h_{l}(t), I=1,2, \ldots, N_{S M}$ is the Rayleigh fading channel coefficient. $n(t) \sim C N\left(0, N_{0}\right)$ expresses a complex Gaussian random process and its variance is $N_{0} / 2$ per dimension.

Because the IQ-components of (1) have a similar structure, the signal model of the proposed system can be rewritten in the terms of only I-component. Thus, at the receiver after the perfect carrier estimation and sapling of the baseband signal, the $l^{\text {th }}$ noisy chip signal received by $r^{\text {th }}$ receive antenna can be expressed as follows:

$r_{j, r}^{l}=x_{I} w_{j_{I}} h_{r, \ell}+n_{j, r}^{l}$,

where, $r=1,2, \ldots, N_{r}$ and $l=1,2, \ldots, L . j$ denotes the code indices of the spreading code. In order to simplify the presentation, the $I$ parameter specifying the $I$-channel is dropped from $r$ and $n$.

As shown by the receiver of the EDAS/COAS-CIM-SM scheme in Figure $1 \mathrm{~b}$, after the despreading operation at the receiver terminal for each IQ-components, each of the branches is used to estimate the spreading code indices, the active antenna indices, 
and the transmitted symbol sequence, i.e., $\left(\hat{J}_{I}, \hat{\jmath}_{Q}, \hat{\ell}_{2}, \hat{x}_{I}, \hat{x}_{Q}\right)$ Here, to reduce the complexity of the EDAS/COAS-CIM-SM system, detection of $\left(\hat{\ell}, \hat{x}_{I}, \hat{x}_{Q}\right)$ parameters will be performed by the EDAS/ COAS based SM detector after the estimation of Walsh Hadamard Code indices $\left(\hat{J}_{I}, \hat{\jmath}_{Q}\right)$ is detected. Therefore, the estimated $\left(\hat{\jmath}_{I}, \hat{\jmath}_{Q}\right)$ indices are fed back to the despreading vector set by using in the selection block diagram in the receiver, and then the $\mathbf{r}_{j i}$ and $\mathbf{r}_{j Q}$ despreading data associated with the $\left(j_{l}, j_{Q}\right)$ are only applied to the input of the EDAS/COAS based SM detector. By this way, the complexity of the system is greatly reduced. For this reason, first, the spreading code indices are estimated from the sampled signals using correlator branches. To do this, the $\mathbf{r}_{j i}$ is multiplied by the corresponding $\mathbf{w}_{j i}$ spreading code in each branch and summed over the period $T_{s}=L T_{c}$. Thus, the despreaded output of the $i^{\text {th }}$ correlator for 1 -component can be expressed as follows:

$$
\begin{aligned}
r_{i, r} & =\sum_{l=1}^{L} w_{i, l} r_{j, r}^{l}=\sum_{l=1}^{L} w_{i, l}\left(x_{I} w_{j_{I}} h_{r, \ell}+n_{j, r}^{l}\right) \\
& = \begin{cases}E_{w} x_{I} h_{r, \ell}+\tilde{n}_{j, r}, & \text { if } i=j \\
\tilde{n}_{j, r}, & \text { if } i \neq j\end{cases}
\end{aligned}
$$

where, $E_{w}$ is the average energy transmitted per spreading code, and it is also expressed as $E_{w}=1 / \sqrt{L} \sum_{l=1}^{L} w_{j, l}^{2} . \tilde{n}_{j, r}=\sum_{l=1}^{L} w_{j, l} n_{j r}$ denotes the additive white Gaussian noise (AWGN) term multiplied by the Walsh Hadamard code. When the despreading operation is performed through all despreader, the resulting vector set at the receiver can be expressed with the help of (3) as follows:

$\psi=\left\{\mathbf{r}_{1}, \mathbf{r}_{2}\right\}$.

In order to estimate the indices of the spreading codes for I-component in the proposed system, first, the quadratic norm of the vectors $\psi=\left\{\left\|\mathbf{r}_{i}^{I}\right\|^{2}\right\}_{i=1}^{N_{c}}$ in the set of $\psi$ is taken, and then, the indices of the maximum element of the squared norm vectors in the sets are determined. Since the Walsh Hadamard Codes are orthogonal to each other, the largest-valued element of the normed vector is also equal to the element over the same indices. From this point forth, the indices of the maximum elements of the normed vector sets for the IQ-components is determined as follows:

$$
\begin{aligned}
\hat{\jmath}_{I} & =\operatorname{argmax}\left\{\left\|\psi^{I}\right\|^{2}\right\}, \\
& =\operatorname{argmax}\left\{\left\|\mathbf{r}_{1}^{I}\right\|^{2},\left\|\mathbf{r}_{2}^{I}\right\|^{2}\right\} \\
\hat{\jmath}_{Q} & =\operatorname{argmax}\left\{\left\|\psi^{Q}\right\|^{2}\right\}, \\
& =\operatorname{argmax}\left\{\left\|\mathbf{r}_{1}^{Q}\right\|^{2},\left\|\mathbf{r}_{2}^{Q}\right\|^{2}\right\} .
\end{aligned}
$$

Once $j_{l}, j_{O}$ are obtained by the spreading code index detectors, EDAS/COAS based SM detector will try all of the combinations of the $\left(\ell, x_{I}\right)$ over the despreaded signals $\mathbf{r}_{j l}$ with $j_{l}$ indices to obtain the estimations of $\left(\hat{\ell}, \hat{x}_{I}\right)$. Since EDAS/COAS based antenna selection technique is used at the transmitter in the CIM-SM system, the detector performance for $\left(\hat{\ell}, \hat{x}_{I}\right)$ parameters is better than traditional SM. Thus, the maximum likelihood (ML) estimation of the $\left(\ell, x_{I}\right)$ parameters for the 1 -component of the proposed EDAS/ COAS-CIM-SM system can be defined as follows:

$$
\left[\hat{\ell}, \hat{x}_{I}\right]=\underset{\substack{\ell \in A=\left\{1,2, \ldots, N_{S M}\right\} \\ X_{I} \in\{-1,+1\}}}{\operatorname{argmin}}\left\{\left\|\mathbf{r}_{j_{I}}^{I}-E_{w} x_{I} \mathbf{h}_{\ell}\right\|^{2}\right\}
$$

where the vector $\mathbf{h}_{l}$ with the size of $N_{r} \times 1$ is the $l^{t h}$ column of the $\mathbf{H}$ channel matrix. The rows of the $\mathbf{H}$ convey the information of the receiving antenna index of the receiver while the columns of the $\mathbf{H}$ carry the information of the active antenna index of the transmitter. The $\mathbf{H}$ can also be written as $\mathbf{H}=\left[\mathbf{h}_{1}\right.$, $\left.\mathbf{h}_{2}, \ldots, \mathbf{h}_{N_{S M}}\right]$.

The other hand, as also shown in Figure $1 \mathrm{~b}$, to reduce the complexity of the receiver $\hat{\ell}$ detected in the I-channel branch is used for the $Q$-channel. Therefore, the ML estimation for $x$ symbol can be expressed as follows:

$$
\hat{x}_{I}=\underset{x_{Q} \in\{-1,+1\}}{\operatorname{argmin}}\left\{\left\|\mathbf{r}_{\hat{\jmath}_{Q}}^{Q}-E_{w} x_{Q} \mathbf{h}_{\hat{\ell}}\right\|^{2}\right\},
$$

Finally, with the bit-back matching technique using the detected $\left(\hat{\jmath}_{I}, \hat{\jmath}_{Q}, \hat{\ell}_{,} \hat{x}_{I}, \hat{x}_{Q}\right)$ values, the originally transmitted bit sequence $\hat{\mathbf{b}}$ is reconstructed at the receiver with help of the demapper as shown in the receiver of the EDAS/COAS-CIM-SM system.

\section{EDAS/COAS Based Spatial Modulation for CIM-SM System}

In this section, EDAS and COAS based SM for CIM-SM system will be presented. As seen in recent studies, antenna selection based wireless communication techniques for MIMO system improves system performance greatly. In these systems, information bits are transmitted through the best antenna related for the channel with the best conditions.

\section{Euclidean Distance optimized Antenna Selection (EDAS) Based SM}

In the traditional SM technique, the information bits are firstly divided into blocks of $\log _{2}\left(N_{t} M\right)$. Then, $\log _{2}\left(N_{t} M\right)$ bits are divided into $\log _{2}\left(N_{t}\right)$ and $\log _{2}(M)$ bits of sub-sequence. Thus, while the $\log _{2}(M)$ bits selects the modulated symbol from the $M$-PSK signal set, on the other hand, $\log _{2}\left(N_{t}\right)$ bits select the $l^{\text {th }}$ transmission antenna out of $N_{t}$. Thus, antennas having good or bad channel conditions will also be activated. From this perspective, in the EDAS or COAS based SM technique, SM scheme is not performed on all the antennas of the transmitting terminal. For example, when considering the EDAS technique on the transmitter, only the number of $N_{S M}$ out of $N_{t}$ transmit antennas will be used for SM technique. To do this, firstly, the set $\wp$ of enumerations of all possible $n=\left(\begin{array}{c}N_{t} \\ N_{S M}\end{array}\right)$ combinations of selecting $N_{S M}$ out of $N_{t}$ transmit antennas will be determined. Then, the best combinations of the set are selected. Later, the SM scheme is performed over these selected antennas 
of the set. Therefore, the transmission data rate is now $\log _{2}\left(N_{S M} M\right)$ bits. For example, if $N_{S M}=2$ transmit antennas out of the $N_{t}=4$ are selected, the $\wp$ set has $n=\left(\begin{array}{l}4 \\ 2\end{array}\right)=6$ elements and these are expressed as $\wp=\{[1,2],[1,3],[1,4],[2,3],[2,4],[3,4]\}$. Among $\left(\begin{array}{c}N_{t} \\ N_{S M}\end{array}\right)$ the possibilities, the specific transmit antenna set which maximizes the minimum Euclidean distance $d_{\min }\left(\mathbf{H}_{x}\right)$ among all possible transmit vectors is defined as follows [8]:

$\chi_{E D}=\underset{\chi \in \wp}{\arg \max }\left\{\min _{\substack{\mathbf{x}_{1}, \mathbf{x}_{2} \in \Omega \\ \mathbf{x}_{1} \neq \mathbf{x}_{2}}}\left\|\mathbf{H}_{\chi}\left(\mathbf{x}_{1}-\mathbf{x}_{2}\right)\right\|^{2}\right\}$,

where $\mathbf{H}_{\chi} \in \mathbb{C}^{\mathrm{N}_{\mathrm{r}} \times N_{S M}}$ has the number of $N_{S M}$ columns determined by $\chi_{E D} . \Omega$ is the set of all possible transmit vectors given by $\Omega=\left\{\mathbf{e}_{\ell} x_{I}\right\}_{\ell=1}^{N_{S M}}$, here, $x_{I} \in\{+1,-1\}$ and $\mathbf{e}_{l}$ presents a vector with size of $N_{S M} \times 1$ having 1 as the only non-zero element at the $l^{\text {th }}$ location, and length of $\Omega$ is the $|\Omega|=N_{S M} M$. If $\mathrm{d}_{\min }(\mathbf{H} x)$ is defined as $d_{\min }\left(\mathbf{H}_{\chi}\right)=\min _{\mathbf{x}_{1}, x_{2} \in \Omega}\left\|\mathbf{H}_{\chi}\left(\mathbf{x}_{1}-\mathbf{x}_{2}\right)\right\|^{2}$, (9) can be rewritten with respect to Euclidean distance as follow:

$\chi_{E D}=\underset{\chi \in \wp}{\arg \max }\left\{d_{\min }\left(\mathbf{H}_{\chi}\right)\right\}$.

Therefore, the system performance can be increased by maximizing $\mathrm{d}_{\min }\left(\mathbf{H}_{x}\right)$. As a result, since the EDAS technique maximizes the $\mathrm{d}_{\text {min }}\left(\mathbf{H}_{x}\right)$, it improves the system performance compared to traditional SM, CIM-SM, CIM-SS schemes. Consequently, the performance of the EDAS-CIM-SM technique is greatly improved when the antenna set $X_{E D}$ obtained in (5) is applied to SM technique.

\section{Capacity Optimized Antenna Selection (COAS) Based SM}

In the case where the channel state information and the signalto-noise ratio (SNR) are known, the capacity of the SM scheme $\left(C_{S M}\right)$ with the $N_{S M}$ transmit antennas can be expressed as follows:

$\beta \leq C_{S M} \leq \beta+\log _{2}\left(N_{S M}\right)$,

where is defined as $\beta=\frac{1}{N_{S M}} \sum_{\ell=1}^{N_{S M}} \log _{2}\left(1+\rho\left\|\mathbf{h}_{\boldsymbol{\ell}}\right\|^{2}\right)$. It can easily be seen from (11) that in order to maximize the capacity of the SM (and thus CIM-SM), the $\beta$ must be maximized. Therefore, the $\beta$ can be maximized by selecting the $N_{S M}$ antennas corresponding to the largest channel norms from the $N_{t}$ transmission antennas. In accordance with these explanations, the set of antenna indices corresponding to $N_{S M}$ largest channel norms out of $N_{t}$ can be calculated in the receiver side, and then, the transmitter is notified back. From this perspective, the COAS based antenna set can be expressed as follows [8]:

$\chi_{C O}=\left\{q_{1}, q_{2}, \ldots, q_{N_{S M}}\right\}$,

The order of $\chi_{C O}$ set is obtained according to the following conditions:

$$
\begin{aligned}
\left\|\mathbf{h}_{q_{1}}\right\|^{2}>\left\|\mathbf{h}_{q_{2}}\right\|^{2}>\cdots>\left\|\mathbf{h}_{q_{N_{S M}}}\right\|^{2}> & \\
& >\left\|\mathbf{h}_{q_{N_{S M}+1}}\right\|^{2}>\cdots>\left\|\mathbf{h}_{q_{N_{t}}}\right\|^{2}
\end{aligned}
$$

Finally, the performance of the COAS-CIM-SM technique is greatly improved when the antenna set $\chi_{C O}$ containing the $\mathbf{h}_{\mathrm{q} 1}, \mathbf{h}_{\mathrm{q} 2}, \ldots, \mathbf{h}_{\mathrm{qN}_{\mathrm{SM}}}$ vectors are used for the SM technique.

\section{Numerical Results}

In this section, Monte Carlo based computer simulation results for the proposed EDAS/COAS-CIM-SM and other compared methods are presented for the M-PSK modulation over the Rayleigh fading channels. In the receiver side, the ML detection technique is performed to obtain the active antenna indices and the transmitted symbols. In order to estimate the spreading code indices of $I Q$-components, correlator method is used. SNR is defined as $\operatorname{SNR}(d B)=10 \log _{10}\left(E_{b} / N_{0}\right)$, here, $E_{b}=\frac{1}{p} \sum_{l=1}^{L}\left(\frac{w_{i, l}}{\sqrt{L}}\right)^{2}$ is the average bit energy and is the number of bits conveyed one symbol. Also, the spreading code is normalized with $\sqrt{L}$ for the transmission power to remain constant and $L=16$. SM, EDAS-SM, COAS SM, CIM-SM, CIM-SS, and DS-SS techniques were used as reference comparisons for the simulation results.

BER performance curves of DS-SS with $N_{t}=1$, 64-PSK; SM with $N_{t}$ $=4$, 16-PSK; CIM-SS with $N_{t}=1$, 4-PSK, $N_{c}^{t}=4$; CIM-SM with $N_{t}=4$, BPSK, $N_{c}=2$; COAS-SM with $N_{t}=6, N_{S M}=4$ 16-PSK; EDAS-SM with $N_{t}=6,{ }_{S M}^{c}=4$, 16-PSK; COAS-CIM-SM with $N_{t}=6, N_{S M}=4, N_{c}=2$, BPSK; and EDAS-CIM-SM with $N_{t}=6, N_{S M}=4, N_{c}=2$, BPSK techniques for $p=6$ bits are depicted when the number of receiver antennas $N_{r}=1$ for Figure 2 and $N_{r}=4$ for Figure 3. The EDAS-CIM-SM, COAS-CIM-SM, and CIM-SM systems transmit 6 bits by 2 bits with antenna indices, 2 bits with spreading code indices, and 2 bits with BPSK symbol via IQ-components. The SM, COAS-SM, and EDAS-SM schemes convey 6 bits: 2 bits antenna indices and 4 bits with a 16PSK modulated symbol. In the CIM-SS technique, 4 bits are carried in spreading code indices and 2 bits are conveyed with the 4-PSK modulated symbol. In the DS-SS scheme, all 6 bits are transmitted on a 64-PSK modulation. Considering Figure 2 and 3, it is seen that the proposed COAS-CIM-SM and EDAS-CIM-SM techniques have a considerable SNR gain compared to other methods.

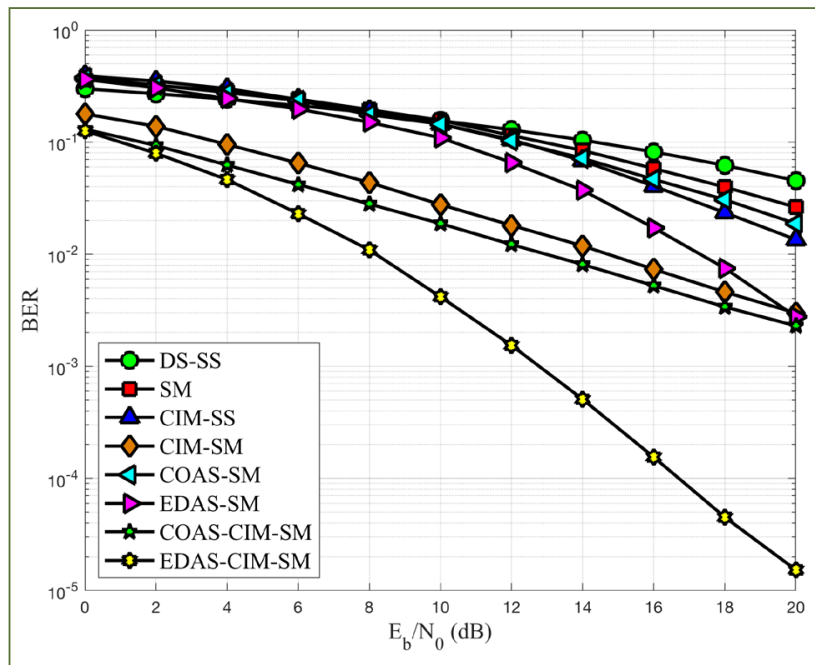

Figure 2. Performance comparison ofDS-SS, SM, CIM-SS, CIM-SM, COASSM, EDAS-SM, COAS-CIM-SM and EDAS-CIM-SM systems for $N_{r}=1$ 


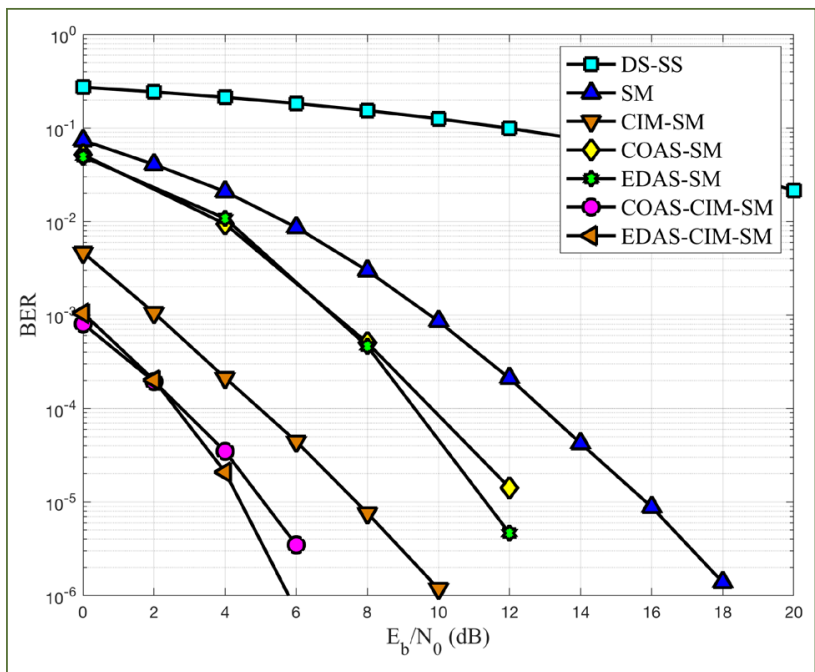

Figure 3. Performance comparison ofDS-SS, SM, CIM-SS, CIM-SM, COASSM, EDAS-SM, COAS-CIM-SM and EDAS-CIM-SM systems for $N_{r}=4$

Figure 4 represents the BER performance comparison curves of DS-SS with $N_{t}=1$, 32-PSK; SM with $N_{t}=2$, 16-PSK; CIM-SM with $N_{t}=2$, BPSK, $N_{c}=2$; COAS-SM with $N_{t}=4, N_{S M}=2$, 16-PSK; EDAS-SM with $N_{t}=4, N_{S M}=2$, 16-PSK; COAS-CIM-SM with $N_{t}=$ $4, N_{S M}=2, N_{c}=2$, BPSK; and EDAS-CIM-SM with $N_{t}=4, N_{S M}=2$, $N_{c}=2$, BPSK techniques for $p=5$ bits are illustrated when the number of receiver antennas $N_{r}=2$.

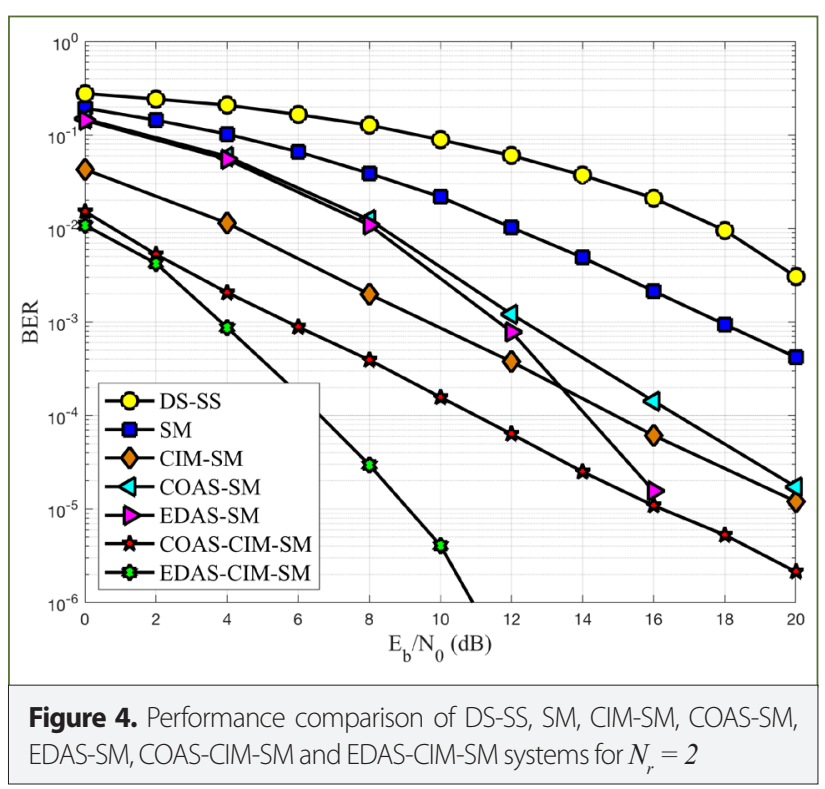

Finally, we can see in Figure 4 that our proposed techniques provide considerable SNR gains compared to other methods for the same number of bits per symbol.

\section{Conclusion}

In this paper, new Euclidian and capacity optimized antenna selection-based MIMO transmission systems with high energy efficiency, high data rate and better error performance, called EDAS-CIM-SM and COAS-CIM-SM, is proposed by combining EDAS, COAS, CIM, and SM techniques which are promising in 5G and beyond communication systems. It is shown via computer simulations that the proposed systems perform data transmission at a higher rate, consumes less transmission energy, and also has better spectral efficiency and performance than the DSSS, CIM-SS, SM, CIM-SM, EDAS-SM and COAS-SM techniques.

Peer-review: Externally peer-reviewed.

Conflict of Interest: The author have no conflicts of interest to declare.

Financial Disclosure: The author declared that the study has received no financial support.

\section{References}

1. C. Patrik. "Ericsson mobility report"White Paper, 2017.

2. S. Talvar, D. Choudhury, K., Dimou, e. Aryafar, b. Bangerter, K. Stewart, "Enabling technologies and architectures for $5 \mathrm{G}$ wireless", IEEE MTT-S International Microwave Symposium (IMS), Tampa, FL, USA, 2014, pp. 1-4. [CrossRef]

3. C. Sasikala, R. Krishnakumar, R., Prabhu, K., Saranya, "A survey of $5 \mathrm{G}$ networks: Design and rising technologies", International Journal of Information and Computing Science, vol. 15, no. 12, pp. 1-7, Dec, 2018.

4. C.Xu, S. Sugiura, S.X.Ng, P.Zhang, L. Wang, L. Hanzo, "Two decades of MIMO design tradeoffs and reduced-complexity MIMO detection in near-capacity systems."IEEE Access, vol. 5, pp. 18564-18632, 2017. [CrossRef]

5. R., Mesleh, A., Abdelhamid, "Space Modulation Techniques,"John Wiley \& Sons, 2018. [CrossRef]

6. S. Sanayei, A. Nosratinia, "Antenna selection in MIMO systems," IEEE Communications Magazine, vol. 42, no. 10, pp. 68-73, Oct, 2004. [CrossRef]

7. T. T. Duman, A. Ghrayeb, "Coding for MIMO communication systems," John Wiley \& Sons, 2008. [CrossRef]

8. R. Rajashekar, K. V. S. Hari, L. Hanzo, "Antenna selection in spatial modulation systems,"IEEE Commun LettVol. 17, no. 3, pp. 521-524, 2013. [CrossRef]

9. P. Narushan, H. Xu, "Comments on antenna selection in spatial modulation systems" IEEE Commun Lett, vol. 17, no. 9, pp. 1681-1683, 2013. [CrossRef]

10. E. Aydin, "Euclidean distance based antenna selection for cooperative spatial modulation", Electrical-Electronics and Biomedical Engineering, Bursa, Turkey, 2018.

11. E. Basar, M, Wen, R. Mesleh, M. Di Renzo, Y. Xiao, H. Haas, "Index modulation techniques for next-generation wireless networks", IEEE Access, vol. 5, pp. 16693-16746, 2017. [CrossRef]

12. M., Wen, X., Cheng, L., Yang, "Index modulation for $5 \mathrm{G}$ wireless communications", Springer, 2017.

13. G. Kaddoum, M.F. A. Ahmed, Y. Nijsure, "Code index modulation: A high data rate and energy efficient communication system", IEEE Commun Lett, vol. 19 no. 2, pp. 175-178, 2015. [CrossRef]

14. G. Kaddoum, Y. Nijsure, H. Tran, "Generalized code index modulation technique for high data rate communication systems", IEEE Trans Veh Technol, vol. 65, no. 9, pp. 7000-7009, 2016. [CrossRef]

15. F. Çögen, E. Aydin, N. Kabaoğlu, E. Basar, H. Ilhan, "A novel MIMO scheme based on code-index modulation and spatial modulation", 26th Signal Processing and Communications Applications Conference (SIU). IEEE, 2018. [CrossRef]

16. F. Çögen, E. Aydin, N. Kabaoğlu, E. Basar, H. Ilhan, "Code index modulation and spatial modulation: A new high rate and energy efficient scheme for MIMO systems." 41st IEEE International Conference on Telecommunications and Signal Processing (TSP), Athens, Greece, 2018. [CrossRef] 
Erdogan Aydın was born in Turkey. He received the B.S. with high honors and the M.S. degrees from Istanbul University, Istanbul, Turkey, in 2007 and 2010, respectively, and the Ph.D. degree from Yıldız Technical University, Istanbul, Turkey, in 2016. From 2007 to 2017, he worked as a Research and Teaching Assistant and Assistant Professor at Istanbul Maltepe University. Since 2011, he has been with the Department of Electronics and Communication Engineering, Istanbul Medeniyet University, Istanbul, Turkey, where He is currently an Assistant Professor. His primary research interests include wireless communications, MIMO Systems, cooperative communication and diversity, Index modulation, spatial modulation, statistical signal processing, and estimation theory. He has received best paper award including one from the IEEE International Conference on Communications 2018. He has served as a TPC member for several IEEE conferences including PIMRC, MENACOMM, AICT and so on. He has authored or coauthored around 17 papers in peer-reviewed journals and conferences. 